\title{
The significance of vortex ring formation to the impulse and thrust of a starting jet
}

\author{
Paul S. Krueger ${ }^{\text {a) }}$ and M. Gharib \\ Graduate Aeronautical Laboratories, California Institute of Technology, Pasadena, California 91125
}

(Received 11 April 2002; accepted 31 January 2003; published 3 April 2003)

The recent work of Gharib, Rambod, and Shariff [J. Fluid Mech. 360, 121 (1998)] studied vortex rings formed by starting jets generated using a piston-cylinder mechanism. Their results showed that vortex rings generated from starting jets stop forming and pinch off from the generating jet for sufficiently large values of the piston stroke to diameter ratio $(L / D)$, suggesting a maximization principle may exist for propulsion utilizing starting jets. The importance of vortex ring formation and pinch off to propulsion, however, rests on the relative contribution of the leading vortex ring and the trailing jet (which appears after pinch off) to the impulse supplied to the flow. To resolve the relative importance of the vortex ring and trailing jet for propulsion, a piston-cylinder mechanism attached to a force balance is used to investigate the impulse and thrust generated by starting jets for $L / D$ ratios in the range $2-8$. Two different velocity programs are used, providing two different $L / D$ values beyond which pinch off is observed, in order to determine the effect of vortex ring pinch off. Measurements of the impulse associated with vortex ring formation show it to be much larger than that expected from the jet velocity alone and proportionally larger than that associated with a trailing jet for $L / D$ large enough to observe pinch off. The latter result leads to a local maximum in the average thrust during a pulse near $L / D$ values associated with vortex rings whose circulation has been maximized. These results are shown to be related to the nozzle exit over-pressure generated during vortex ring formation. The over-pressure is in turn shown to be associated with the acceleration of ambient fluid by vortex ring formation in the form of added and entrained mass.

(C) 2003 American Institute of Physics. [DOI: 10.1063/1.1564600]

\section{INTRODUCTION}

Starting jets can be described as a jet or pulse issuing into quiescent fluid. An interesting flow feature that has long been associated with starting jets is the vortex ring, the formation of which is due largely to the roll up of the jet shear layer as it is ejected (see Didden ${ }^{1}$ ). Indeed, investigations of the formation and evolution of vortex rings produced by starting jets occupy a substantial body of recent literature (e.g., Didden, ${ }^{1}$ Nitsche and Krasny, ${ }^{2}$ Maxworthy, ${ }^{3}$ Auerbach, ${ }^{4}$ Glezer, ${ }^{5}$ Glezer and Coles, ${ }^{6}$ James and Madnia, ${ }^{7}$ and the review by Shariff and Leonard ${ }^{8}$ ). Nature, on the other hand, uses starting jets as a basic component in a remarkable variety of flows, ranging from jet pulses entering the human left ventricle to the pulsatile propulsion of aquatic creatures such as squid and salps. This apparent preference for unsteady jet flows in nature leaves one to question whether or not there is something utilitarian about the formation of vortex rings in starting jets over and above their current position in fluid mechanics as simple, yet rich, flow structures. Of particular interest to this investigation is the relationship between vortex ring formation and the impulse supplied to the flow by individual starting jets. A clear understanding of this relationship has application to pulsatile propulsion and synthetic jet flows, to name a few.

a) Present address: Department of Mechanical Engineering, Southern Methodist University, P.O. Box 750337, Dallas, TX 75275.
The idea that vortex ring formation might play a fundamental role in certain natural processes or be useful in mechanical devices was brought to the forefront by the recent work of Gharib, Rambod, and Shariff ${ }^{9}$ (GRS). GRS used a piston-cylinder mechanism to generate starting jets by translating a piston of diameter $D$ through a length $L$. For sufficiently small $L / D$ (i.e., less than about 4 ), they observed individual vortex rings, as expected. For larger $L / D$, however, the leading vortex ring stopped entraining energy and circulation from the generating jet, resulting in a flow characterized by a leading vortex ring and a trailing jet. For such cases, the leading vortex ring is said to have "pinched off" from the generating jet. The value of the nondimensional, time-varying piston displacement, $X(t) / D$, at which pinch off occurred was dubbed the "formation number," $F$. For the cases tested by GRS, $F$ was found to be between 3.6 and 4.5, suggesting it has a relatively universal value. Later numerical work, however, showed that the formation number could be increased by steadily increasing the piston velocity, $U_{p}(t)$, during the stroke (Rosenfeld et al. ${ }^{10}$ and Mohseni et al. ${ }^{11}$ ), or by increasing the nozzle diameter during pulse ejection (Mohseni et al. ${ }^{11}$ ). Rosenfeld et al. ${ }^{10}$ also showed that blunting the jet velocity profile at the nozzle exit plane to a parabolic profile could reduce the formation number to approximately 1 .

Despite the susceptibility of vortex ring pinch off to manipulation, the existence of the formation number is still interesting in that it represents a maximization principle for 
vortex ring formation. (Indeed, the analysis by GRS and the later work by Mohseni and Gharib ${ }^{12}$ firmly related vortex ring pinch off to the idea that beyond a certain point, the vortex generating mechanism was no longer able to supply energy consistent with the requirement that the vortex ring has maximal energy with respect to impulse-preserving, isovortical perturbations.) GRS were the first to recognize the practical significance of this maximization principle, suggesting it might imply optimal pulsing conditions for cardiac flows or for the pulsatile propulsion of certain aquatic creatures. While this suggestion at first seems intuitive, it relies on the assumption that the leading vortex ring in some sense dominates the flow. For example, in the case of propulsion it is known that even for $L / D \gg F$, the trailing jet still contributes thrust. So, the hypothesis that vortex ring pinch off (and hence, the formation number) is significant for propulsion relies on the relative significance of the leading vortex ring to the trailing jet for generating impulse.

It is the primary goal of this investigation to address the relative significance of the leading vortex ring and trailing jet for propulsion. In particular, we wish to determine if vortex ring pinch off leads to an optimum in some propulsive quantity (such as impulse or thrust) and if so, what features of vortex ring formation are responsible for the propulsive benefit provided. These issues are approached experimentally by measuring the thrust and impulse generated by starting jets over a range of $L / D$ for two piston velocity programs. The resulting flow is also characterized using digital particle image velocimetry (DPIV) and hotfilm measurements of the jet velocity.

\section{EXPERIMENTAL SETUP}

A layout schematic of the relevant features of the apparatus used to generate the starting jets for this experiment is shown in Fig. 1. The basic system consists of two pistoncylinder arrangements (one oriented vertically and the other oriented horizontally) submerged in water and connected by a combination of PVC piping and a flexible hose. Because of the incompressibility of water, the floating piston in the horizontal cylinder follows the motion of the driver piston (actuated by the servo motor), as indicated by the gray arrows in Fig. 1. The horizontal cylinder is mounted to a force balance to measure the thrust and total impulse generated by the starting jets. The force balance was custom designed with a stiffness in the direction of the jet axis of $5.07 \times 10^{5} \mathrm{~N} / \mathrm{m}$ and a force resolution of better than $0.0098 \mathrm{~N}$ in the absence of any signal conditioning. To minimize the effect of the forming vortex ring's interaction with the nozzle on the force measurements, a sharp cone angle of $7^{\circ}$ was used at the nozzle exit, as shown in Fig. 2. Figure 2 also defines the cylindrical coordinate system used for this experiment and the jet exit velocity, $u_{J}(r, t)$.

The entire apparatus was mounted in a tank facility with dimensions of $373 / 4 \mathrm{in} .(95.9 \mathrm{~cm})$ by $333 / 4 \mathrm{in} .(85.7 \mathrm{~cm})$ by approximately $12 \mathrm{ft} 31 \mathrm{in.}(444.5 \mathrm{~cm})$. The tank frame was steel and the walls were $3 / 4$ in. glass for flow visualization purposes. The apparatus was rigidly fixed to the top of the tank with steel fixtures. The hatch marks in Fig. 1 indicate

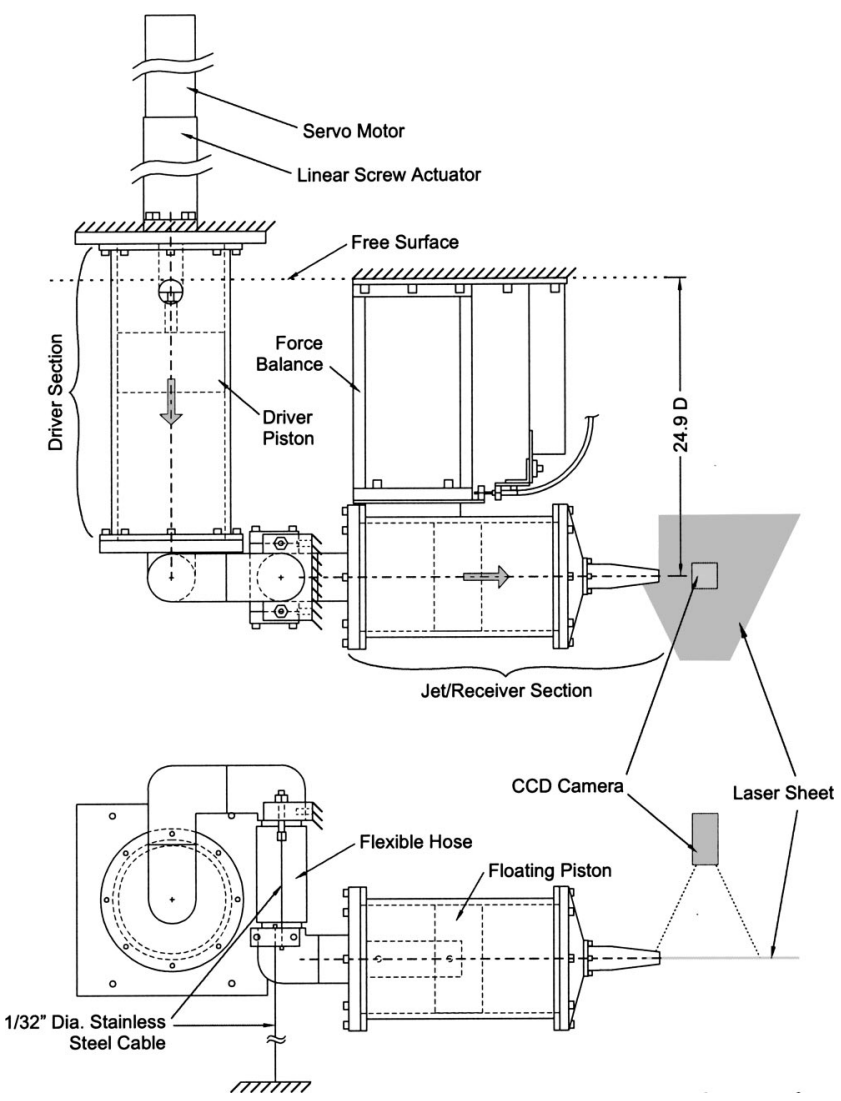

FIG. 1. Schematic of the apparatus used to generate a starting jet. Only those hidden features necessary to illustrate the operation of the device are shown. Hatch marks indicate those features which have been rigidly attached to the tank.

the portions of the apparatus that were, in some way, rigidly fixed to the tank. The entire apparatus was mounted at one end of the tank, making the minimum separation of the nozzle from any of the boundaries that of the distance between the nozzle center line and the free surface, namely 12.45 in. $(31.6 \mathrm{~cm}=24.9 D$ where $D=0.500$ in. is the nozzle diameter).

A laser sheet and CCD were mounted as shown in Fig. 1 to make DPIV measurements of the flow (see Willert and

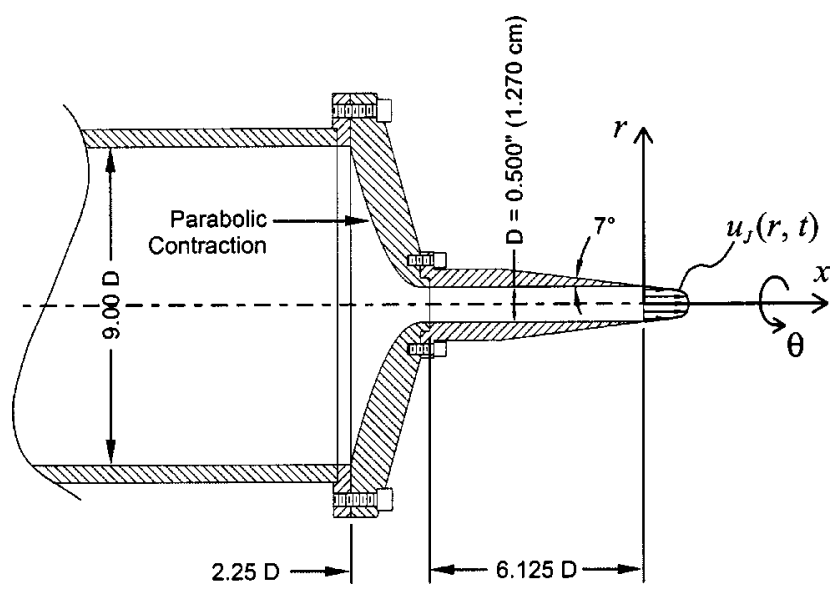

FIG. 2. Cross section of the jet nozzle with a definition of the cylindrical coordinate system used in this investigation. 


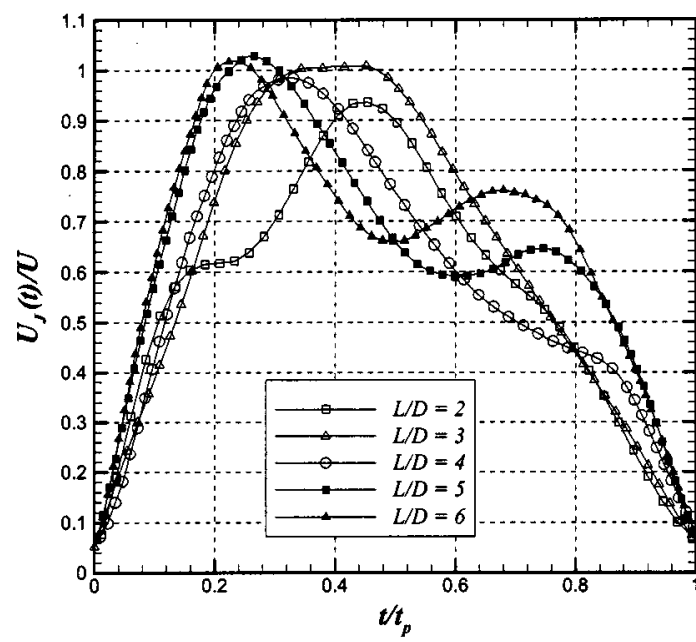

(a)

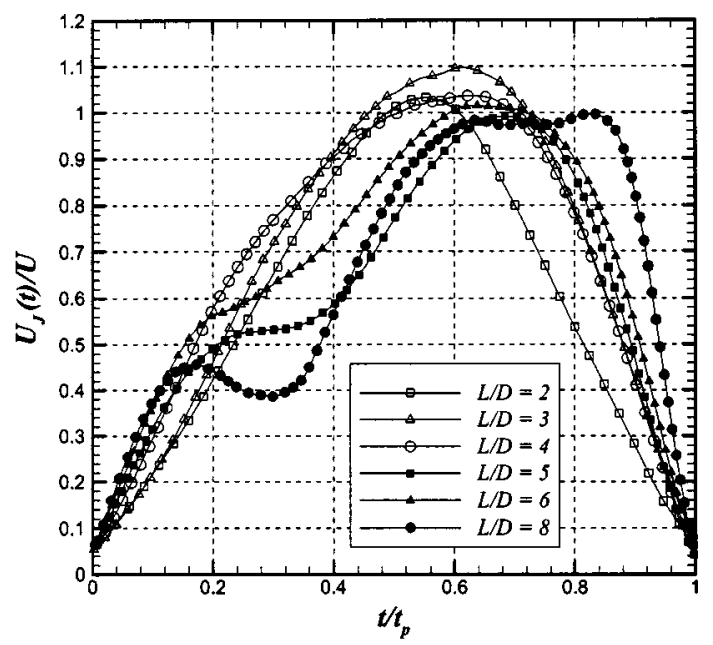

(b)

FIG. 3. The velocity programs used to generate the starting jets: (a) negative sloping (NS) ramps, (b) positive sloping (PS) ramps.

Gharib ${ }^{13}$ and Raffel et al. ${ }^{14}$ ). For DPIV measurements, the flow was seeded with neutrally buoyant, silver coated hollow glass spheres with diameters in the range of $20-50 \mu \mathrm{m}$. The particles were illuminated with an Nd:YAG laser whose beam was formed into a 2-mm-thick sheet using a cylindrical lens. Recording the images on a $480 \times 768 \mathrm{CCD}$ and using a window shifting algorithm (see Westerwheel et al. ${ }^{15}$ ) to cross-correlate the images with a $32 \times 32$ interrogation window at $50 \%$ overlap produced flow vector fields with a spatial resolution of $0.099 D$ in $x$ and $0.12 D$ in $r$. This setup is nearly identical to that used by GRS, giving results accurate to within $1 \%$ in velocity and $3 \%$ in vorticity.

Hotfilm measurements of the jet velocity at the nozzle exit plane $(x=0)$ were accomplished with a TSI $1231 \mathrm{~W}$ hotfilm probe using a TSI IFA-100 flow analyzer and a model 150 anemometer. The probe was placed at the jet center line and calibrated to the time-averaged flow rates, $\overline{U_{J}}$, produced by commanding the driver piston to move at several steady velocities, $U_{s}$. From continuity, $\overline{U_{J}}$ and $U_{s}$ are related by

$$
\overline{U_{J}}=\left(\frac{D_{p}}{D}\right)^{2} U_{s}
$$

where $D_{p}$ is the driver piston diameter. Here $U_{J}(t)$ is defined by

$$
U_{J}(t) \equiv \frac{1}{A} \int_{A} u_{J}(r, t) d S,
$$

where $A$ is the cross-sectional area of the nozzle exit and $u_{J}(r, t)$ is jet velocity at $x=0$ (see Fig. 2). Fitting a curve to the results gave a calibration curve that fell within $2 \%$ of the known calibration values over the range of velocities observed in this investigation. An independent calibration for

$$
r_{v}=\frac{\overline{U_{J}}(r=0)}{\overline{U_{J}}} \equiv \overline{\overline{u_{\mathrm{cl}}}}
$$

was obtained using DPIV measurements of the timeaveraged center line velocity, $\overline{u_{\mathrm{cl}}}$, for several steady com- manded piston velocities. Fitting a curve to $r_{v}$ as a function of $U_{J}$ gave a calibration curve for $r_{v}$ that agreed with the calibration values to within $2 \%$ over the range of velocities considered in this investigation. These two calibration curves allowed determination of $U_{J}(t)$ and $u_{\mathrm{cl}}(t)$ from the hotfilm measurements at $x=0, r=0$. [That the time-varying behavior of $U_{J}(t)$ could be determined for the velocity programs considered (see Fig. 3) from a steady-state calibration was confirmed by comparing the expected results from the calibration with those calculated from the model formulated by Atabek and Chang ${ }^{16}$ for unsteady entrance pipe flow.]

\section{EXPERIMENTAL PARAMETERS}

The functional form of $U_{J}(t)$ is called the velocity program for a starting jet. As noted earlier, the velocity program can have a significant effect on the formation number $F$, so this experiment utilized two classes of velocity programs for a range of $L / D$. Hotfilm anemometry measurements of the velocity programs for each $L / D$ in each class (averaged over 20 realizations) are shown in Fig. 3. The coordinates of Fig. 3 are $t / t_{p}$ and $U_{J}(t) / U$, where $U$ is the desired peak velocity of $1.03 \mathrm{~m} / \mathrm{s}$ and $t_{p}$ is the pulse duration. The velocity programs in Fig. 3(a) are primarily negative sloping in the sense that $U_{J}(t)$ is decreasing through the majority of the pulse, so this class of velocity programs is called the negative sloping (NS) ramps. Conversely, the velocity programs in Fig. 3(b) are primarily positive sloping and are called the PS ramps. (Although the velocity programs do not collapse exactly for all $L / D$ in each case due to a small amount of elasticity between the driver and floating pistons, the overall form of the velocity programs in each class is as desired.) Following Rosenfeld et al., ${ }^{10}$ the Reynolds number for these velocity programs is defined as

$$
\operatorname{Re}_{m} \equiv \frac{U_{\max } D}{\nu},
$$

where $U_{\max }$ is the maximum $U_{J}$ achieved during a pulse. The measurements in Fig. 3 indicate that $\operatorname{Re}_{m}$ was 13000 to 


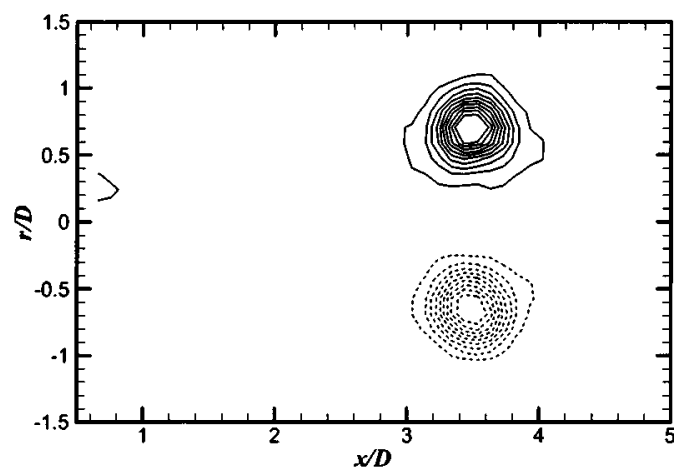

(a) $L / D=2.0$, NS Ramp

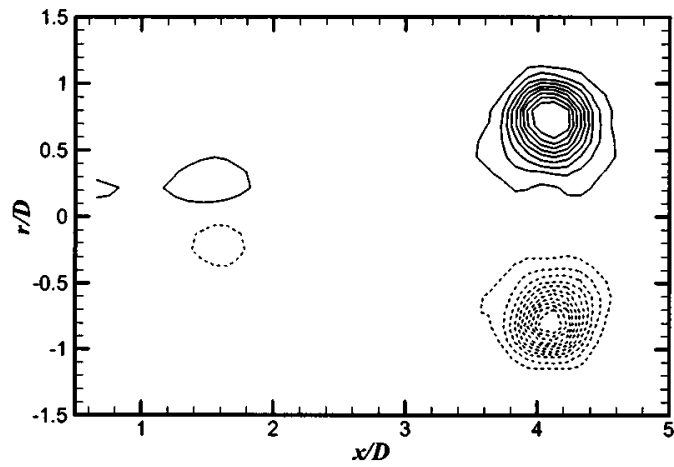

(b) $L / D=3.0$, NS Ramp

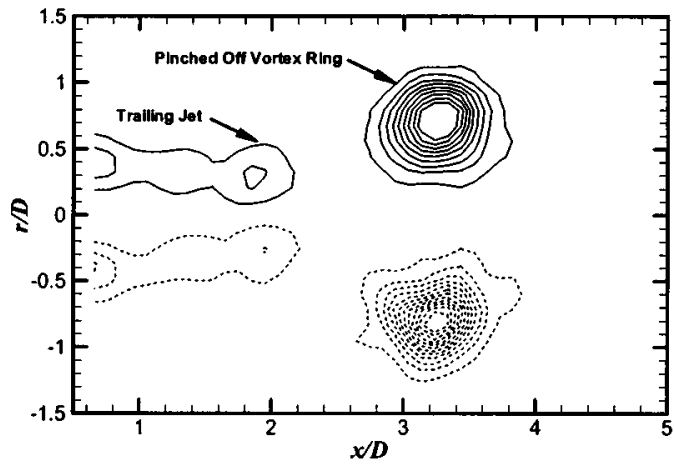

(c) $L / D=4.0$, NS Ramp

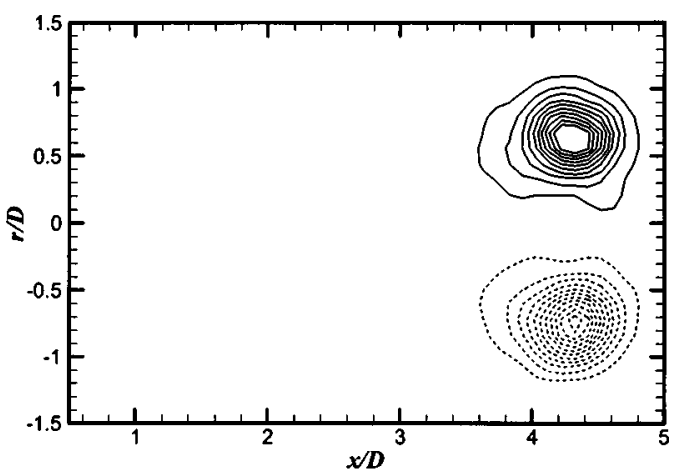

(d) $L / D=3.0$, PS Ramp

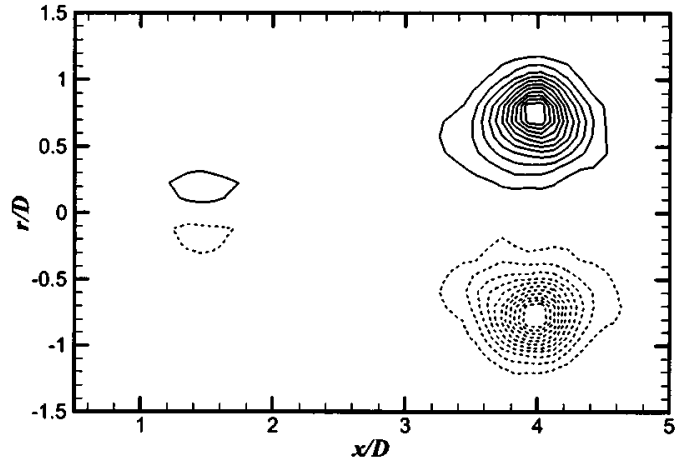

(e) $L / D=4.0$, PS Ramp

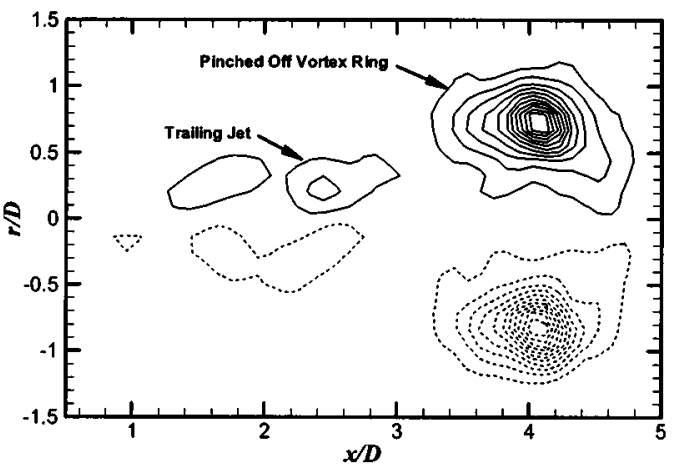

(f) $L / D=5.0$, PS Ramp

FIG. 4. DPIV measurements of the vorticity for the NS and PS ramps over a range of $L / D$. The solid contours are positive vorticity and the dashed contours are negative vorticity. The magnitude of the lowest contour is $301 / \mathrm{s}$ (positive or negative). Notice that the same progression of events takes place with increasing $L / D$ for both the NS and PS ramps, but the sequence for the PS ramps is shifted by $+1 L / D$ relative to the NS ramps.

within $10 \%$ for the NS and PS ramps. Finally, it follows from Eq. (2) that the stroke ratio for starting jets used in this experiment is given by

$$
\frac{L}{D} \equiv \frac{1}{D} \int_{0}^{{ }^{t} p} U_{J}(\tau) d \tau=\frac{L_{p}}{D}\left(\frac{D_{p}}{D}\right)^{2},
$$

where $L_{p}$ is the displacement of the driver piston during a pulse. The last equality in Eq. (5) follows from continuity.

The form of the NS and PS classes of velocity programs was chosen to take advantage of the effect observed by Rosenfeld et al. ${ }^{10}$ that the formation number can be significantly increased by using a velocity program with a steadily increasing velocity. In particular, these velocity programs were designed so that the $L / D$ for which a significant trailing jet appeared would be higher for the PS ramps than the NS ramps. This effect is illustrated graphically in Fig. 4, which shows DPIV measurements of vorticity for $L / D=2,3,4$ of the NS ramps and $L / D=3,4,5$ of the PS ramps. Figures 4(a)-4(c) show that the NS ramps produce isolated vortex rings for $L / D \leqslant 3$, but at $L / D=4$, the leading vortex ring has pinched off from the generating jet and a significant trailing jet appears. The sequence of events for the PS ramps is very similar [as illustrated in Figs. 4(d), 4(e), and 4(f)], but the sequence is shifted by $+1 L / D$ relative to the NS ramps so 
TABLE I. Global parameters for the experimental conditions considered.

\begin{tabular}{cccccc}
\hline \hline \multirow{2}{*}{$\begin{array}{c}L / D \\
\text { (nominal) }\end{array}$} & \multicolumn{2}{c}{$\operatorname{Re}_{\Gamma}($ ring) } & & \multicolumn{2}{c}{ Formation number $(F)^{\mathrm{b}}$} \\
\cline { 2 - 3 } \cline { 5 - 6 } \cline { 5 - 6 } & NS ramp & PS ramp & & NS ramp & PS ramp \\
\hline 2 & $1.5 \times 10^{4}$ & $1.8 \times 10^{4}$ & & $\mathrm{NPO}^{\mathrm{c}}$ & $\mathrm{NPO}^{\mathrm{c}}$ \\
3 & $2.2 \times 10^{4}$ & $2.4 \times 10^{4}$ & & $2.43^{\mathrm{d}}$ & $\mathrm{NPO}^{\mathrm{c}}$ \\
4 & $2.4 \times 10^{4}$ & $2.7 \times 10^{4}$ & & 2.71 & $3.31^{\mathrm{d}}$ \\
5 & $2.4 \times 10^{4}$ & $2.8 \times 10^{4}$ & & 2.88 & 4.12 \\
6 & - & - & & 3.04 & 4.37 \\
8 & \multicolumn{3}{c}{-} & & 4.94 \\
\hline \hline
\end{tabular}

${ }^{a}$ Reynolds number of the vortex ring or pinched-off vortex ring (as appropriate), namely, $\operatorname{Re}_{\Gamma} \equiv \Gamma / \nu$ where $\Gamma$ is the circulation of the vortex ring and $\nu$ is the kinematic viscosity. The ring circulation was determined using DPIV. DPIV data were taken for $L / D \leqslant 5$ only. The precision of the results fall within $0.1 \times 10^{4}$

${ }^{\mathrm{b} C a l c u l a t e d}$ from the model proposed by Shusser-Ref. 22 (see Sec. V A). The uncertainty is typically less than 0.10 for the cases studied.

${ }^{\mathrm{c}}$ No pinch-off. The formation number is only listed for cases where a trailing jet (of any size) can be distinguished.

dThe trailing "jet" is very weak in these cases. A trailing jet does not become substantial until higher $L / D$ (see Fig. 4).

that a significant trailing jet does not appear for the PS ramps until $L / D=5$. Thus, if vortex ring formation and pinch off is critical to the thrust and impulse produced by starting jets, measurements of these quantities as a function of $L / D$ should show a shift of about $+1 L / D$ between the NS and PS ramps.

Having established these two classes of velocity programs and their effect on vortex ring pinch off, the total impulse (time integral of the measured thrust) was measured for 25 pulses at each $L / D$ of the velocity programs shown in Fig. 3. The velocity programs for the NS and PS ramps were measured using hotfilm anemometry for 20 pulses before and after the force measurements were taken. Finally, DPIV measurements of the pinched-off vortex rings were made for 10 pulses with $2 \leqslant L / D \leqslant 5$ (both NS and PS ramps) where the downstream viewing area of the camera was $0.45<x / D$ $<5.25$. The relatively large number of trials for each measurement allowed for ensemble averaging to reduce noise and other sources of variation. The error bars in the follow- ing results represent the standard deviation of the mean of the respective measurements, indicating the precision or repeatability of the results with this setup (the accuracy having been established by the accuracy of the measurement techniques and their calibration). A summary of the cases considered, including Reynolds numbers $(\Gamma / \nu)$ and formation numbers of the pinched-off vortex rings, is provided in Table I.

\section{IMPULSE AND THRUST MEASUREMENTS}

Measurements of the total impulse per pulse were accomplished for each of the velocity programs depicted in Fig. 3 by calculating

$$
I=\int_{0}^{\infty} F_{\mathrm{FB}}(\tau) d \tau
$$

where $F_{\mathrm{FB}}$ is the force measured by the force balance. ${ }^{17}$ The ensemble averages of the measurements for each case are shown in Fig. 5. The solid reference lines in Fig. 5 are matched to the slopes of the first few points in each case ( $L / D \leqslant 3$ for the NS ramps and $L / D \leqslant 4$ for the PS ramps). The dashed reference lines are matched to the slopes of the total impulse due to jet momentum flux, $I_{U}$, at $L / D \geqslant 4$ for the NS ramps and $L / D \geqslant 5$ for the PS ramps. For this axisymmetric case, $I_{U}$ is given by

$$
I_{U} \equiv I_{U}\left(t_{p}\right)=\rho \int_{0}^{t_{p}} \int_{A} u_{J}^{2}(r, \tau) d S d \tau .
$$

If in the nozzle, $u_{J}$ is assumed to be constant in a core region $0 \leqslant r \leqslant \delta$ and parabolic for $\delta \leqslant r \leqslant D / 2$ [following Prandtl (see Prandtl and Tietjens ${ }^{18}$ )], then $I_{U}$ can be expressed entirely in terms of $U_{J}(t)$ and $r_{v}$ ( $\delta$ can be eliminated).${ }^{19}$ This idea was used to calculate $I_{U}$ from the hotfilm measurements of the velocity programs, thereby giving the slopes of the dashed lines shown in Fig. 5. Comparison of this method for determining $I_{U}$ with the predictions of the model for unsteady pipe flow by Atabek and Chang ${ }^{16}$ showed it to be accurate to within $5 \%$.

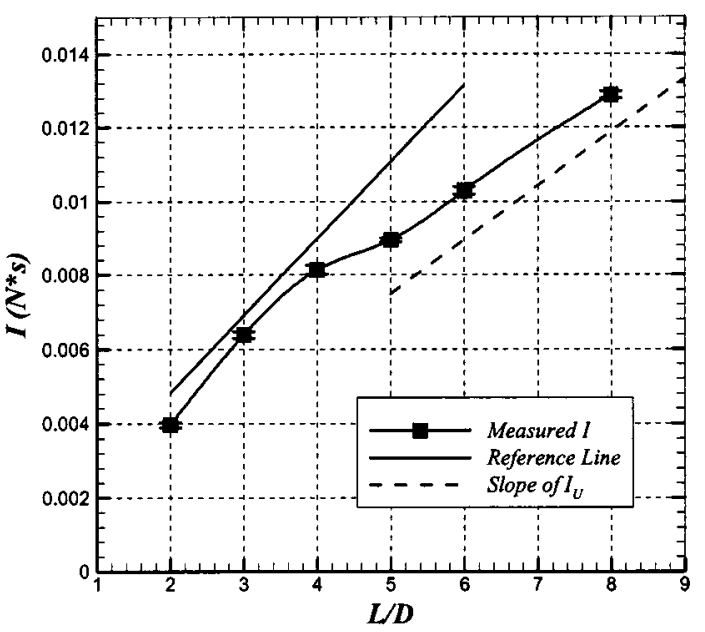

(b)

(a)

FIG. 5. Total impulse (per pulse) for the velocity programs depicted in Fig. 3: (a) NS ramps, (b) PS ramps. 


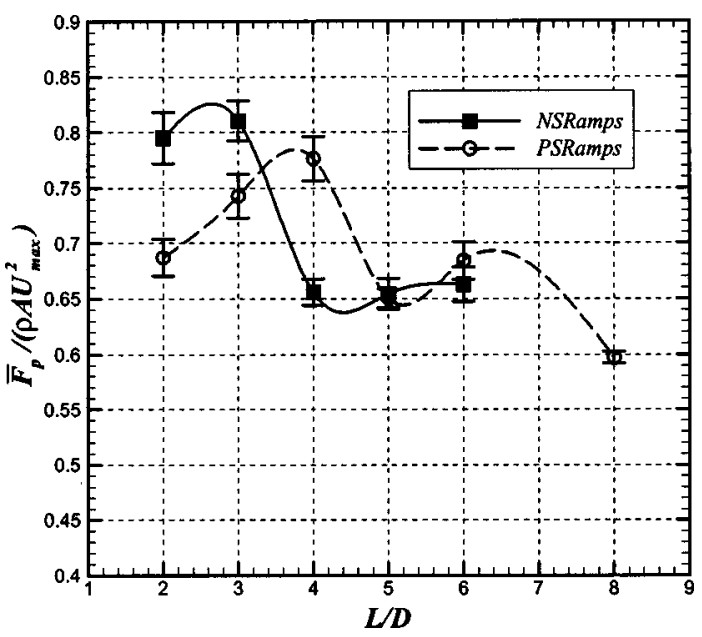

FIG. 6. Normalized average thrust per pulse measurements.

The total impulse measurements in Fig. 5 show the total impulse follows a generally increasing trend with $L / D$, which is to be expected since the jet is on for a longer period of time for larger $L / D$. On closer inspection, however, a transition is seen at $L / D=3$ for the NS ramps and $L / D=4$ for the PS ramps when the measurements are compared with the solid reference lines. Specifically, the impulse increases at a lower rate with increasing $L / D$ for $L / D>3$ in the NS case and $L / D>4$ in the PS case. This represents a shift in the transition point of $L / D=1$ between the NS and PS cases, following the observed shift in the trailing-jet growth seen in Fig. 4. Furthermore, comparing Fig. 5 with Fig. 4 shows that the rate of increase of total impulse with $L / D$ changes where a trailing jet becomes a significant part of the flow. This implies that vortex ring formation plays a significant role in determining the impulse of a starting jet. Indeed, for $L / D$ $>3$ and $L / D>4$ of the NS and PS ramps, respectively, $I$ increases at nearly the same rate as the impulse from a steady jet (shown by the dashed reference line for the slope of $I_{U}$ ). The difference in slopes between the solid and dashed reference lines therefore indicates the difference between a "vortex ring-phase" and "jet-phase," demonstrating that a trailing jet contributes less to the total impulse per unit $L / D$ than a comparable increase in the strength of a leading vortex ring.

While the total impulse measurements lend great insight into the relative importance of the leading vortex ring and trailing jet for generating impulse, it is helpful to account for the fact that the duration of the pulse increases with $L / D$. This is addressed by determining the average thrust during a pulse, $\overline{F_{p}}$, defined as

$$
\overline{F_{p}} \equiv \frac{I}{t_{p}},
$$

where $t_{p}$ is the pulse duration. Figure 6 shows $\overline{F_{p}}$ normalized by $\rho A U_{\max }^{2}$ and plotted as a function of $L / D$ for the NS and PS ramps.

The results for $\overline{F_{p}} /\left(\rho A U_{\text {max }}^{2}\right)$ clearly show a peak in this quantity near $L / D=4$ for the PS ramps. A similar trend is apparent for the NS ramps with a peak occurring at $L / D$ $=3$ in this case, but this peak is not as well defined because the values for $L / D=2$ and 3 are very similar. Nevertheless, a sharp decrease in $\overline{F_{p}} /\left(\rho A U_{\max }^{2}\right)$ with increasing $L / D$ is clearly apparent at $L / D=3$ for the NS ramps. The locations of the peaks in $\overline{F_{p}} /\left(\rho A U_{\max }^{2}\right)$ are very close to the abovenoted $L / D$ values where a significant trailing jet begins to appear and are shifted to higher $L / D$ for the PS ramps as expected from Fig. 4. These facts suggest a direct link between a maximum in $\overline{F_{p}} /\left(\rho A U_{\max }^{2}\right)$ and vortex ring pinch off. [The link is less pronounced for the NS ramps because the maximum is not as sharp as for the PS ramps, but it is clear that pinch off is associated with a sharp decrease in $\overline{F_{p}} /\left(\rho A U_{\max }^{2}\right)$ as $L / D$ is increased.] That is, $\overline{F_{p}} /\left(\rho A U_{\max }^{2}\right)$ is maximized by pulses near the conditions that generate vortex rings of maximum circulation without leaving a trailing jet. Stated differently, once the $L / D$ of a pulse is large enough that the leading vortex ring has pinched off and a trailing jet is present, an additional increase in $L / D$ will provide more impulse, but it takes proportionally more time to do it than if the additional fluid was actually added to the forming vortex ring.

Overall the impulse and thrust measurements directly indicate the importance of the formation of a leading vortex ring in starting jets for impulse and thrust generation, showing that a trailing jet plays a less important role for these quantities. In particular, the average thrust per pulse measurements demonstrate that a propulsive quantity maximized by formation of a vortex ring near the conditions of maximal circulation and a minimal trailing jet is $\overline{F_{p}} /\left(\rho A U_{\max }^{2}\right)$.

\section{NOZZLE EXIT OVER-PRESSURE AND ITS CONNECTION TO VORTEX RING FORMATION}

While the results of Sec. IV clearly answer the question of the importance of vortex ring formation and pinch off for the dynamic quantities $I$ and $\overline{F_{p}} /\left(\rho A U_{\max }^{2}\right)$, the source of the benefit provided by vortex ring formation over that of the trailing jet is unclear. The difficulty lies in the fact that the standard model used for vortex ring formation is the slug model, which makes no distinction between the vortex ring and the trailing jet (see Krueger ${ }^{20}$ Shusser and Gharib, ${ }^{21}$ Mohseni and Gharib ${ }^{12}$ ). It is therefore necessary to consider a more accurate description of vortex ring formation in order to address the issue of the impulse benefit provided by the formation of a vortex ring.

Through a control volume analysis of the fluid region external to a piston-cylinder mechanism, it can be shown (with minimal assumptions) that the impulse, energy, and circulation injected into the flow by a starting jet are determined by a flux term and a contribution from over-pressure at the nozzle exit plane (nozzle exit over-pressure) $\left(\right.$ Krueger $\left.^{20}\right)$. Specifically, the result for impulse is

$$
I(t)=I_{U}(t)+I_{p}(t),
$$

where $I_{U}(t)$ is defined in Eq. (7) and $I_{p}(t)$, called the pressure impulse, is defined by

$$
I_{p}(t) \equiv \int_{0}^{t} \int_{A}\left[p(r, \tau)-p_{\infty}\right] d S d \tau .
$$




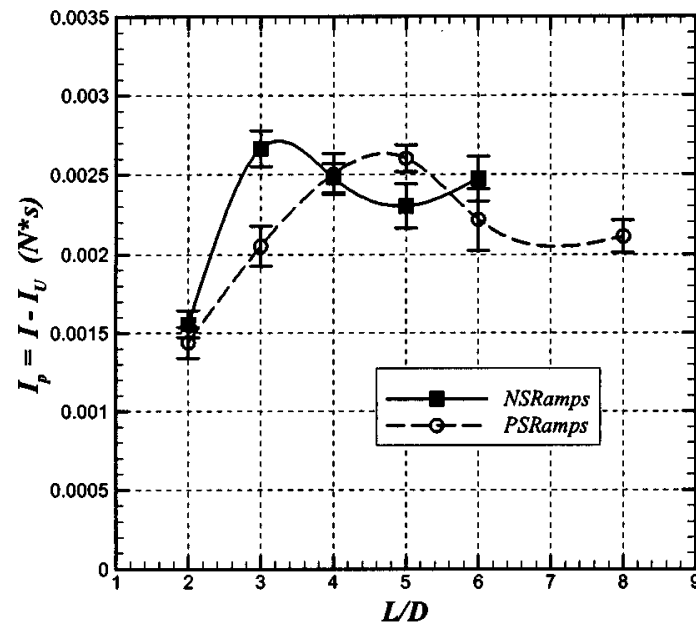

(a)

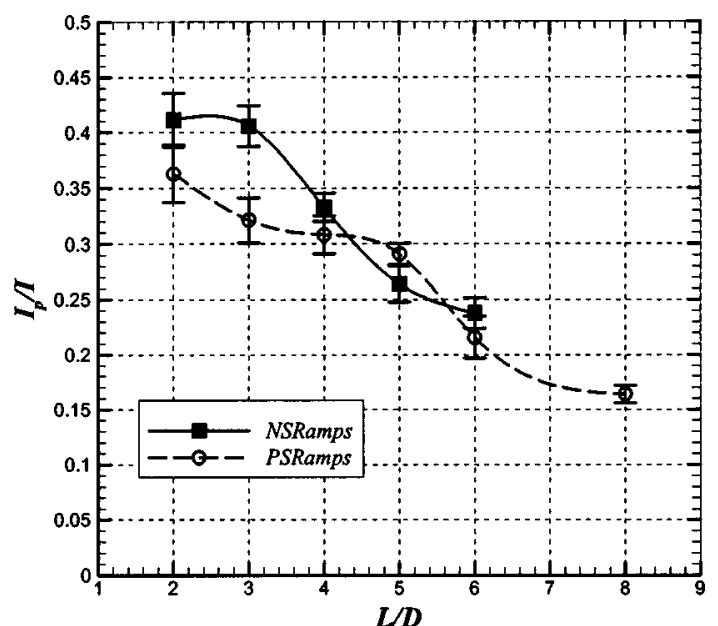

(b)

FIG. 7. Pressure contribution to impulse for the NS and PS ramps: (a) pressure impulse, (b) normalized pressure impulse.

In Eq. (10), $p_{\infty}$ is defined as the ambient pressure at $(x, r)$ $\rightarrow(\infty, 0)$. The slug model, along with most of the literature on vortex rings, ignores the pressure contribution to vortex ring formation. Here it is demonstrated that the propulsive benefit provided by the vortex ring over that of the trailing jet is determined by nozzle exit over-pressure during vortex ring formation, which can be related to the acceleration of ambient fluid by the forming vortex ring.

\section{A. The pressure contribution to impulse}

Equation (9) gives the total pressure impulse per pulse as $I_{p}=I-I_{U}$,

where $I$ is determined from the measurements of the total impulse and $I_{U}$ can be determined from the measurements of the jet velocity during a pulse. For the NS and PS ramp cases studied here, Eq. (11) gives the results shown in Fig. 7(a). The significance of the pressure contribution is illustrated in Fig. 7(b) where the fraction of $I$ contributed by $I_{p}$ is plotted. As before, the error bars represent the precision of the measurements.

Figure 7(a) shows that the pressure impulse increases with $L / D$ for small $L / D$, but for sufficiently large $L / D$ (i.e., $L / D>3$ for the NS ramps and $L / D>4$ for the PS ramps), the dependence of $I_{p}$ on $L / D$ is very weak, showing a slight decline. Since a trailing jet does not become a significant part of the flow until $L / D>3$ for the NS ramps and $L / D>4$ for the PS ramps, it follows that the pressure impulse increases only as long as the vortex ring continues to develop. Indeed, the weak dependence of $I_{p}$ on $L / D$ for larger $L / D$ motivates the approximation $I_{p}(t) \approx 0$ for $t>t_{F}$, or more boldly, $p(r, t) \approx p_{\infty}$ for $t>t_{F}$ where $t_{F}$ can be called the "formation time" and denotes the time during the pulse at which the circulation (eventually) entrained by the ring has been ejected. That is, nozzle exit over-pressure is ignored during the ejection of a trailing jet. Such an approximation makes sense physically as long as there is no sudden acceleration of the jet for $t>t_{F}$ so that the trailing jet can be treated as a "steady" jet. Nearly all of the velocity programs studied ex- hibit this behavior. The only exception is the $L / D=6.0$ case of the NS ramps where a second peak in the velocity program is observed around $t / t_{p}=0.75$ [see Fig. 3(a)].

In addition to the observations drawn from Fig. 7(a), Fig. 7(b) indicates that the pressure impulse makes a very significant contribution to the total impulse, reaching as much as $42 \%$ for the NS ramp at the lower $L / D$ values. The slug model would, therefore, seriously underestimate the total impulse (especially for small $L / D$ ) since it ignores this pressure term.

Figure 7(b) also illustrates an important difference between the NS and PS ramps. While the trends for both velocity programs are similar (with the trend for the PS ramps being shifted to a higher $L / D$ ), the values of $I_{p} / I$ tend to be lower for the PS ramps. This difference is especially apparent for smaller $L / D$. Clearly the velocity program has a significant effect on the pressure impulse over and above its effect on the formation number. Similar observations can be made about $\overline{F_{p}} /\left(\rho A U_{\max }^{2}\right)$ from Fig. 6 , but the results for $I_{p} / I$ seem more enlightening. In particular, the values of $I_{p} / I$ at $L / D=2.0$ are very nearly the same for the NS and PS ramps. This is because the velocity programs for NS and PS ramps are very similar at this small $L / D$ (see Fig. 3). Conversely, the differences in $I_{p} / I$ for the NS and PS cases at larger $L / D$ must be related to the more gradual initiation of the PS ramps.

Returning to the above-mentioned approximation that $I_{p}(t) \approx 0$ for $t>t_{F}$, a quantitative test of this approximation can be made with a model of vortex ring pinch off appropriate for the cases studied here. Such model had been proposed by Shusser ${ }^{22,23}$ as an extension of the ideas in Shusser and Gharib $^{21}$ to time-varying piston velocity programs. It is based on the hypothesis that vortex ring pinch off occurs when the ring velocity exceeds the jet velocity in the immediate vicinity of the ring. Using analytical approximations for the jet and ring velocity allows implicit determination of $t_{F}$ for a specified velocity program. Then the formation number is calculated as 


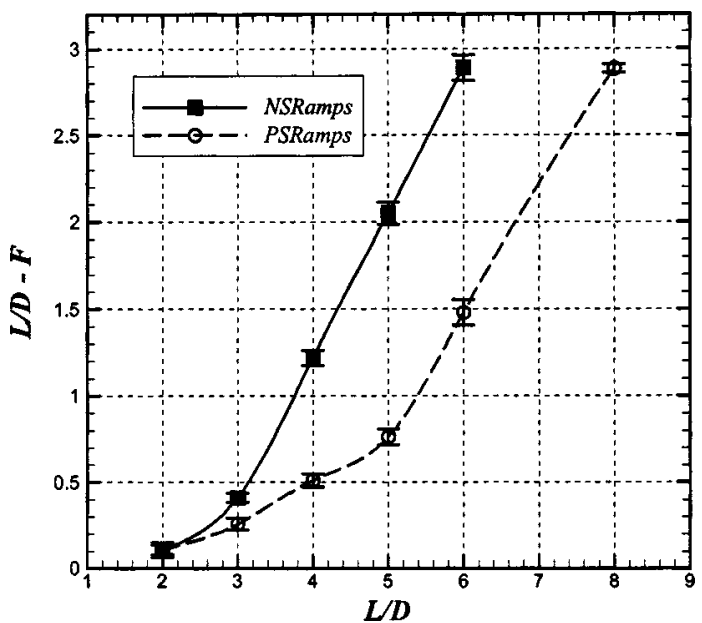

FIG. 8. Nondimensional trailing jet length $L / D-F$ for the velocity programs used in this experiment (calculated using the model of ShusserRef. 22).

$$
F=\frac{1}{D} \int_{0}^{t_{F}} U_{J}(\tau) d \tau
$$

When no pinch off occurs, this model predicts $L / D=F$ so that $L / D-F$ is a measure of the nondimensional trailing jet length when $F$ is calculated from Eq. (12). For the velocity programs used in this experiment, the model gives the values of $L / D-F$ shown in Fig. 8. These results agree remarkably well with Fig. 4 in that a significant trailing jet (i.e., $L / D-F>0.5)$ is predicted for $L / D>3$ of the NS ramps and $L / D>4$ of the PS ramps, indicating the model gives a reliable $t_{F}$ for these velocity programs. (Unfortunately it was impossible to do a more precise comparison of the model predictions with the actual formation number since the time resolution of DPIV is insufficient to allow direct determination of the formation number for the NS and PS ramps.)

Using the model proposed by Shusser ${ }^{22}$ to determine $t_{F}$ and the above-noted approximation that $I_{p}(t) \approx 0$ for $t>t_{F}$, the impulse due to the trailing jet can be approximated as

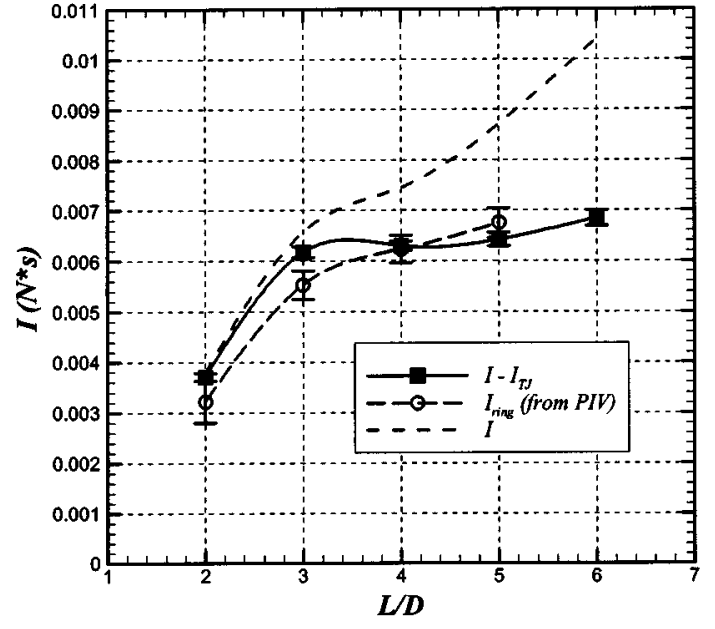

(a)

$$
I_{\mathrm{TJ}} \approx \rho \int_{t_{F}}^{t_{p}} \int_{A} u_{J}^{2}(r, \tau) d S d \tau .
$$

With this approximation, the impulse contributed by the formation of the leading vortex ring alone is calculated as

$$
I_{\text {ring }}=I-I_{\mathrm{TJ}} \text {. }
$$

Applying Eqs. (13) and (14) to the impulse measurements gives the results shown in Fig. 9. Figure 9 also gives the impulse of the leading vortex ring determined from PIV measurements of the vorticity field, where, for axisymmetric flow, the impulse of the ring is given by

$$
I_{\text {ring }}=\pi \rho \int_{\text {ring }} \omega_{\theta}(r, x) r^{2} d S
$$

(Lim and Nickels ${ }^{24}$ ). In Eq. (15), $\rho$ is the fluid density, $\omega_{\theta}$ is the azimuthal component of vorticity, $r$ is the radial coordinate (see Fig. 2), and the integration is over the vorticity associated with the ring in a plane containing the nozzle centerline.

Two conclusions follow from a comparison of the approximated ring impulse shown in Fig. 9 with the total measured impulse $I$ (plotted as the dashed reference line) and the ring impulse measured from PIV. First, the approximated ring impulse determined using Eqs. (14) and (13) starts to level off for $L / D>3.0$ for the NS ramps and $L / D>4.0$ for the PS ramps, as expected from vortex ring pinch off. Second, the approximated ring impulse agrees with the measured ring impulse to within $10 \%$ in all cases (for $L / D$ $<5.0$ ) and $8 \%$ in most cases. The later result gives strong confirmation of the assumption that nozzle exit over-pressure adds a trivial amount of impulse after the vortex ring pinches off from the generating jet. Stated differently, the trailing jet can be approximated as a steady jet.

The apparent lack of nozzle exit over-pressure during the ejection of a trailing jet and the significant over-pressure provided during vortex ring formation indicates that the propulsive benefit provided by vortex ring formation is determined by nozzle exit over-pressure. The need for over-pressure is

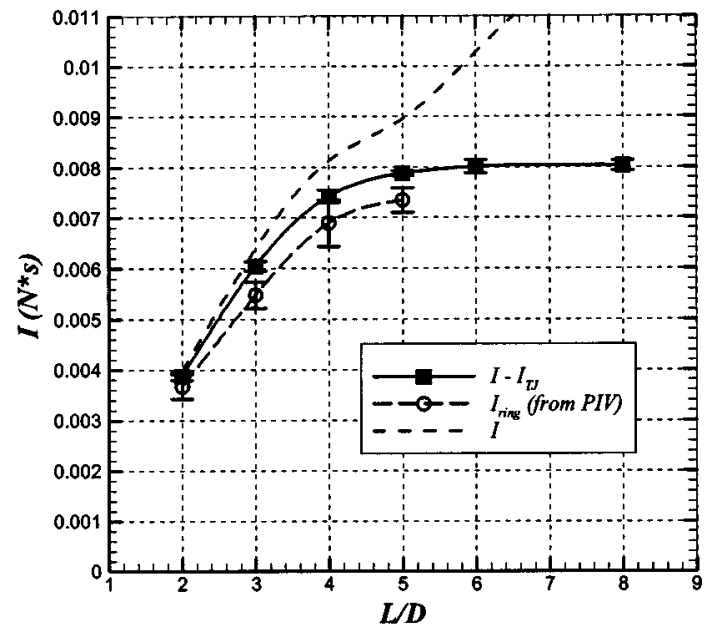

(b)

FIG. 9. Measurements of the ring impulse obtained from total impulse measurements and PIV vorticity measurements: (a) NS ramps, (b) PS ramps. 


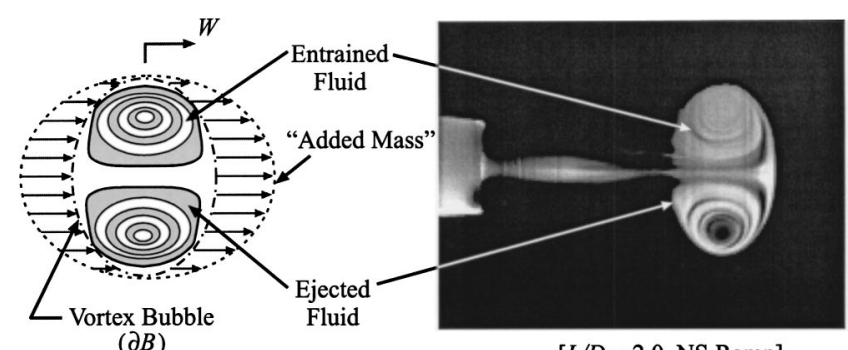

$(\partial B)$

$[L / D=2.0$, NS Ramp $]$

FIG. 10. Illustration of the three classes of ambient fluid accelerated by a vortex ring. (The image on the right is PLIF flow visualization for $L / D$ $=2.0, \mathrm{NS}$ ramp.)

related to the acceleration of ambient fluid (i.e., the inertia of the ambient fluid) during the ejection of a starting jet, giving the jet something to push off of as it is ejected. This can be illustrated in a rather novel way by considering the entrained ambient fluid and added mass associated with an axistouching vortex ring.

\section{B. The origin of nozzle exit over-pressure as expressed through added and entrained mass}

Consider an isolated, axis-touching vortex ring as shown in the planar laser induced fluorescence (PLIF) image on the right in Fig. 10. The flow associated with the vortex ring can be conceptually divided into three different masses of moving fluid, as illustrated schematically on the left in Fig. 10. The first is the fluid ejected from the nozzle ( $\left.m_{\text {ejected }}\right)$, shown as the dyed fluid in the PLIF image. The second is entrained fluid ( $\left.m_{\text {entrained }}\right)$, which appears as dark bands in the PLIF image of the vortex ring in Fig. 10. Entrained fluid, as defined here, is ambient fluid that has been entrained into the vortex ring bubble (defined as the dyed region of fluid, $\partial B$ ) as the shear layer from the nozzle boundary layer rolls up into a ring near the nozzle exit (Didden $\left.{ }^{1}\right)$. Entrained fluid moves downstream at the mean velocity of the ring. The third component of moving fluid is added mass $(M)$, which is ambient fluid set into motion as the ring forms because some of the fluid in front of the jet must be accelerated out of the way when the starting jet is initiated and some ambient fluid must be brought in behind the ring to preserve continuity of the flow once it begins moving downstream. The illustration of this on the left in Fig. 10 is meant to convey the added mass associated with the motion of a completely formed vortex ring (i.e., the $M$ associated with the vortex ring bubble $\partial B)^{25,26}$

For an isolated vortex ring, the momentum of these three components of the fluid motion completely account for the impulse required to generate the flow and hence, for the impulse (thrust) felt by the vortex ring generator. That is, it follows from hydrodynamics (see the Appendix) that the hydrodynamic impulse associated with an isolated vortex ring can be expressed as

$$
I_{v}=\left(m_{\text {ejected }}+m_{\text {entrained }}+M\right) W,
$$

where $W$ is the velocity of the vortex $\operatorname{ring}, M$ is the added mass of the vortex bubble $\partial B$, and $m_{\text {ejected }}$ and $m_{\text {entrained }}$ were defined earlier. For $L / D$ sufficiently small so that no trailing jet is formed, $I_{v}$ also represents the total impulse in the flow, $I$, which by Eq. (11) is equivalent to $I_{U}+I_{p}$ and by Newton's third law is equivalent to the total impulse measured by the force balance. Thus,

$$
I=I_{U}+I_{p}=\left(m_{\text {ejected }}+m_{\text {entrained }}+M\right) W
$$

for an isolated vortex ring.

To interpret Eq. (17), it is helpful to note that the momentum of the ejected fluid $\left(m_{\text {ejected }} W\right.$ ) is derived from the jet momentum, i.e., from $I_{U}$, since the jet was comprised of this fluid. In fact, from experiments it follows that $I_{U}$ $>m_{\text {ejected }} W$ for vortex rings with no trailing jet, ${ }^{27}$ so none of $m_{\text {ejected }} W$ is contributed by $I_{p}$. (The fact that $I_{U}>m_{\text {ejected }} W$ indicates that some of the jet momentum has been distributed to other components of the fluid motion, such as $m_{\text {entrained }} W$.) Therefore, $I_{p}$ is only associated with $\left(m_{\text {entrained }}\right.$ $+M) W$. Stated differently, $I_{p}$ contributes only to the acceleration of ambient fluid in the form of added and entrained mass from rest to a velocity of $W$. Because the contribution to $I_{p}$ from a trailing jet is negligible, this conclusion also holds if a trailing jet is present (even though the calculation of $M$ is complicated by the presence of the trailing jet).

Taking these observations together with the previous discussion of the pressure impulse measurements demonstrates that the primary benefit of a leading vortex ring over a trailing jet for producing thrust or impulse is its mechanisms for moving and entraining ambient fluid. One contribution of this perspective is that it suggests modeling added and entrained mass contributions to the flow during ring development may provide a simple approach for analytical evaluation of $I_{p}(t)$. Such modeling would require extending the above-noted ideas to a developing ring (as opposed to a developed ring). A rudimentary model for the added mass contribution at pulse initiation was proposed by Krueger ${ }^{20}$ where the shape of the starting jet at its initiation was approximated as a disk. Much more work is required, however, to account for entrainment and the growth of the ring during the rollup of the shear layer.

While the concepts of added and entrained mass add physical insight into the flow associated with the pressure impulse, the present data and analysis leave some unresolved issues. First, the above-presented data and analysis center on $I_{p}$ where what is actually of interest is $I_{p}(t)$. Some insight into $I_{p}(t)$ can be achieved by looking at $I_{p}$ as a function of $L / D$, but the precise development of pressure impulse as a function of time cannot be resolved by the present experimental setup since it only measures per-pulse quantities. Detailed knowledge of $I_{p}(t)$ would be useful for the abovedescribed modeling efforts as well as for illuminating the differences in $I_{p} / I$ between the NS and PS ramps. Second, the inequality $I_{U}>m_{\text {ejected }} W$ for vortex rings indicates that $I_{U}$ is also associated with $M$ and $m_{\text {entrained }}$. As a consequence, conclusions about entrainment or added mass cannot be made directly from $I_{p}$. What is needed are direct measurements of $m_{\text {entrained }}$ and $M$, which are not available from the present experimental results. Despite these unresolved issues, the concepts of added and entrained mass clarify important points and motivate further analysis/modeling. 


\section{CONCLUSIONS}

Our experimental studies show that a forming vortex ring contributes proportionally more impulse per unit $L / D$ than a trailing jet, demonstrating the dominant role played by vortex rings for generating impulse and underscoring the importance of vortex ring pinch off. Normalizing the results by the pulse duration, $t_{p}$, to determine the (normalized) average thrust during a pulse, $\overline{F_{p}} /\left(\rho A U_{\max }^{2}\right)$, showed that this propulsive quantity is optimized by starting jets at $L / D$ just after pinch off is observed (although the optimum is less pronounced for the NS ramps), demonstrating that vortex ring pinch off represents an optimization principle for $\overline{F_{p}} /\left(\rho A U_{\max }^{2}\right)$. While more research is needed to determine the relevance of these results to pulsatile aquatic propulsion or cardiac flows, it is clear that they highlight the importance of vortex ring formation and pinch off for generating impulse, suggesting design strategies for pulsed-jet technologies whose primary goal is to impart impulse to the flow (such as synthetic jets).

The important role played by vortex ring formation in the thrust and impulse of starting jets was related to nozzle exit over-pressure. It was found that over-pressure supplied additional impulse only during vortex ring formation and provided essentially no effect during the ejection of a trailing jet. Furthermore, the pressure impulse contribution to the total impulse was found to be as much as $42 \%$ of the total impulse for cases involving isolated vortex rings. This leads to the conclusion that nozzle exit over-pressure must be included in models of vortex ring formation from a pistoncylinder mechanism, a point on which the slug model is wholly inadequate. Indeed, the slug model only appears to be valid during the ejection of a trailing jet.

The physics of the pressure impulse for vortex ring formation were related to the acceleration of ambient fluid in the form of added and entrained mass using a hydrodynamic analysis of a completely developed ring. While this analysis gives a nice perspective that may be useful in future modeling attempts for $I_{p}$, it does not resolve the relative importance of the acceleration of entrained and added mass to $I_{p}$. It also does not resolve how $I_{p}$ develops as a pulse is ejected. This is an important point worthy of further consideration, both for the purpose of designing pulsed jet devices and for modeling vortex ring formation. For instance, it is expected that more detailed information would shed light on to the difference in $I_{p} / I$ for the NS and PS ramps. For the moment, the authors hypothesize that this difference is related to added mass effects at pulse initiation, but measurements of $I_{p}$ as a function of time are needed to confirm this. In any case, it is hoped that this work has brought the importance of nozzle exit over-pressure and its relationship to vortex ring formation to the forefront of research in this area.

\section{ACKNOWLEDGMENT}

The authors would like to acknowledge the Powell Foundation for partial support of this work.

\section{APPENDIX: IMPULSE OF AN ISOLATED VORTEX RING}

The hydrodynamic impulse, $\mathbf{I}_{v}$, of a vortex bound by a material surface $\partial B$ can be expressed as

$$
\mathbf{I}_{v}=\mathbf{P}_{B}+\mathbf{I}_{a},
$$

where

$$
\mathbf{P}_{B} \equiv \rho \int_{B} \mathbf{u} d V
$$

and $\mathbf{I}_{a}$ is the virtual momentum of the material surface (see Saffman, ${ }^{28}$ Sec. 4.2). The physical meaning of $\mathbf{I}_{v}$ is that it is the impulse that must be applied to the fluid to generate the flow. The virtual momentum, $\mathbf{I}_{a}$, is the impulse required to set a (massless) body in the shape of $\partial B$ in motion against the inertia of the fluid.

For a vortex ring, $\partial B$ is defined as the largest stream surface in the frame of reference moving with the ring velocity $W$ that contains all of the ring vorticity. This is equivalent to the vortex bubble illustrated in Fig. 10. Since the flow velocity on $\partial B$ in the frame moving with the ring is tangential to $\partial B$, it follows from the vector identity

$$
\int_{B} \mathbf{u} d V=-\int_{B} \mathbf{x}(\boldsymbol{\nabla} \cdot \mathbf{u}) d V+\int_{\partial B} \mathbf{x}(\mathbf{u} \cdot \hat{\mathbf{n}}) d S
$$

and the incompressibility of the flow (i.e., $\boldsymbol{\nabla} \cdot \mathbf{u}=0$ ) that

$$
\mathbf{P}_{B}=\rho V_{B} W \hat{\mathbf{x}},
$$

where $V_{B}$ is the volume of the vortex ring bubble and $\hat{\mathbf{x}}$ is aligned with the axis of the ring. [The vector identity (A3) is valid for any differentiable vector field $\mathbf{u}$, where $\hat{\mathbf{n}}$ is the out-ward unit normal to $\partial B$ (see Appendix $\mathrm{B}$ of $\mathrm{Wu}$ and $\mathrm{Wu}^{29}$ ).] For a thick cored ring (i.e., an axis-touching ring), $B$ is simply connected and $\mathbf{I}_{a}$ is given by

$$
\mathbf{I}_{a}=\lambda \rho V_{B} W \hat{\mathbf{x}},
$$

where $M=\lambda \rho V_{B}$ is the added mass of the vortex bubble. ${ }^{30-32}$ That is, $\lambda$ is given by

$$
\lambda=\frac{1}{V_{B}} \int_{\partial B} \Phi \frac{\partial \Phi}{\partial n} d S,
$$

where $\phi=W \Phi$ satisfies $\nabla^{2} \phi=0$ with the boundary condition $\partial \phi / \partial n=W(\hat{\mathbf{x}} \cdot \hat{\mathbf{n}})$ on $\partial B$ (see Sec. 5.1 of $\mathrm{Saffman}^{28}$ ). Given that $V_{B}=V_{\text {ejected }}+V_{\text {entrained }}$ as illustrated by Fig. 10, it follows that

$$
I_{v}=\left(m_{\text {ejected }}+m_{\text {entrained }}+M\right) W,
$$

where $m_{\text {ejected }}$ and $m_{\text {entrained }}$ are the mass of the ejected fluid and the mass of the fluid entrained into the vortex bubble, respectively.

\footnotetext{
${ }^{1} \mathrm{~N}$. Didden, "On the formation of vortex rings: Rolling-up and production of circulation," Z. Angew. Math. Phys. 30, 101 (1979).

${ }^{2} \mathrm{M}$. Nitsche and R. Krasny, "A numerical study of vortex ring formation at the edge of a circular tube," J. Fluid Mech. 276, 139 (1994).

${ }^{3}$ T. Maxworthy, "Some experimental studies of vortex rings," J. Fluid Mech. 81, 465 (1977).

${ }^{4} \mathrm{D}$. Auerbach, "Experiments on the trajectory and circulation of the starting vortex," J. Fluid Mech. 183, 185 (1987).

${ }^{5}$ A. Glezer, "The formation of vortex rings," Phys. Fluids 31, 3532 (1988).
} 
${ }^{6} \mathrm{~A}$. Glezer and D. Coles, "An experimental study of a turbulent vortex ring," J. Fluid Mech. 221, 243 (1990).

${ }^{7}$ S. James and C. K. Madnia, "Direct numerical simulation of a laminar vortex ring," Phys. Fluids 8, 2400 (1996).

${ }^{8}$ K. Shariff and A. Leonard, "Vortex rings," Annu. Rev. Fluid Mech. 24, 235 (1992)

${ }^{9}$ M. Gharib, E. Rambod, and K. Shariff, "A universal time scale for vortex ring formation," J. Fluid Mech. 360, 121 (1998).

${ }^{10}$ M. Rosenfeld, E. Rambod, and M. Gharib, "Circulation and formation number of laminar vortex rings,” J. Fluid Mech. 376, 297 (1998).

${ }^{11} \mathrm{~K}$. Mohseni, H. Ran, and T. Colonius, "Numerical experiments on vortex ring formation," J. Fluid Mech. 430, 267 (2001).

${ }^{12} \mathrm{~K}$. Mohseni and M. Gharib, "A model for universal time scale of vortex ring formation," Phys. Fluids 10, 2436 (1998).

${ }^{13}$ C. E. Willert and M. Gharib, "Digital particle image velocimetry," Exp. Fluids 10, 181 (1991).

${ }^{14}$ M. Raffel, C. E. Willert, and J. Kompenhans, Particle Image Velocimetry: A Practical Guide (Springer, Berlin, 1998).

${ }^{15}$ J. Westerweel, D. Dabiri, and M. Gharib, "The effect of a discrete window offset on the accuracy of cross-correlation analysis of digital PIV recordings," Exp. Fluids 23, 20 (1997).

${ }^{16} \mathrm{H}$. B. Atabek and C. C. Chang, "Oscillatory flow near the entry of a circular tube," Z. Angew. Math. Phys. 12, 185 (1961).

${ }^{17}$ The convention used throughout this paper is that $I$ (without reference to time dependence) refers to the total impulse per pulse.

${ }^{18}$ L. Prandtl and O. G. Tietjens, Applied Hydro- and Aeromechanics (Dover, New York, 1934).

${ }^{19}$ The short duration of the pulses made it difficult to directly check this assumption using DPIV. However, the DPIV results for steady commanded piston velocities show a velocity distribution very similar to the assumed profile. Furthermore, the large $\mathrm{Re}_{m}$ used for these experiments ensures the velocity profile is nearly uniform over most of the nozzle diameter, so the exact shape of the velocity profile in the boundary layer has only a small effect on the calculation of $I_{U}$.

${ }^{20} \mathrm{P}$. S. Krueger, "The significance of vortex ring formation and nozzle exit over-pressure to pulsatile jet propulsion," Ph.D. thesis, California Institute of Technology, 2001.

${ }^{21}$ M. Shusser and M. Gharib, "Energy and velocity of a forming vortex ring," Phys. Fluids 12, 618 (2000).

${ }^{22}$ It has been suggested to the authors by M. Shusser that the model of Shusser and Gharib (Ref. 21) can be applied to the cases studied in the present manuscript by making the following modifications: (a) The ring properties as a function of time are determined from $\Gamma(t)$ $=\frac{1}{2} \int_{0}^{t} U_{J}^{2}(\tau) d \tau, I(t)=\rho A \int_{0}^{t} U_{J}^{2}(\tau) d \tau$, and $E(t)=\rho A / 2 \int_{0}^{t} U_{J}^{3}(\tau) d \tau$, where $\Gamma, I$, and $E$ are the circulation, impulse, and kinetic energy of the ring, respectively. These equations are used as opposed to the slug model results for constant piston velocity used in Shusser and Gharib (Ref. 21). (b) The nondimensional thickness of the ring core, $\epsilon$, is taken as 0.4 at vortex ring pinch off. A justification for selection of this value is provided by Shusser et al. (Ref. 23). Using these modifications, the vortex ring velocity, the ring radius, and the jet velocity near the ring can be determined as described in Shusser and Gharib-Ref. 21 (see also Shusser et al.-Ref. 23). Applying the criterion that pinch off occurs when the ring velocity surpasses the velocity of the jet near the ring (Shusser and Gharib-Ref. 21) gives an implicit equation for the formation time $t_{F}$, which yields the formation number using Eq. (12). This methodology was used to compute the formation numbers for the velocity programs in Fig. 3 and generate Fig. 8.

${ }^{23}$ M. Shusser, M. Gharib, M. Rosenfeld, and K. Mohseni, "On the effect of pipe boundary layer growth on the formation of a laminar vortex ring generated by a piston/cylinder arrangement," Theor. Comput. Fluid Dyn. 15, 303 (2002).

${ }^{24}$ T. T. Lim and T. B. Nickels, "Vortex rings," in Fluid Vortices, edited by S. I. Green (Kluwer Academic, New York, 1995).

${ }^{25}$ The illustration of the added mass effect in Fig. 10 suggests the added mass of the fluid external to the ring is actually convected with the ring in the sense that the same fluid particles are always in front of and behind the ring. Since the vorticity in the ring generates a velocity field that tends to sweep fluid around the boundary of the ring, this cannot be entirely true. Indeed, when a starting jet is initiated, this effect is manifested as ambient fluid getting pushed out of the way. It is therefore not possible to define this added mass as entrained mass in the traditional sense. The fluid inducted into the body of the ring, however, can be considered entrained mass in that it is convected downstream with the ring.

${ }^{26}$ Perhaps a term other than "added mass" should be used to describe the potential flow external to a completely formed vortex ring since it is associated with the steady motion of the ring while added mass is typically related to accelerating bodies. Since, however, it is the acceleration of the forming ring that leads to the motion of this fluid, added mass as used here also refers to the fluid motion external to a completely formed vortex ring.

${ }^{27}$ For example, the results for $L / D=2$ of the NS ramps are $m_{\text {ejected }} W$ $=1.34 \times 10^{-3} \pm 0.09 \times 10^{-3} \mathrm{~N} \mathrm{~s}$ and $I_{U}=2.23 \times 10^{-3} \pm 0.04 \times 10^{-3} \mathrm{~N} \mathrm{~s}$.

${ }^{28}$ P. Saffman, Vortex Dynamics (Cambridge University Press, Cambridge, 1992).

${ }^{29}$ J. Z. Wu and J. M. Wu, "Interactions between a solid surface and a viscous compressible flow field," J. Fluid Mech. 254, 183 (1993).

${ }^{30}$ If the vortex ring is thin enough that the vortex "bubble" is actually a torus (as assumed by Weihs-Ref. 31 and Miloh et al.-Ref. 32), then an extra term is required in Eq. (22) to correct for the fact that the "bubble" is not simply connected (see Saffman-Ref. 28-pp. 81, 200).

${ }^{31}$ D. Weihs, "Periodic jet propulsion of aquatic creatures," Fortschr. Zool. 24, 171 (1977).

${ }^{32}$ T. Miloh, G. Waisman, and D. Weihs, "The added-mass coefficients of a torus," J. Eng. Math. 12, 1 (1978). 